\title{
Characterizing Trabecular Bone Properties near the Glenohumeral Joint Following Brachial Plexus Birth Injury
}

Emily B. Fawcett ${ }^{\mathrm{a}, 1}$, Carolyn M. McCormick ${ }^{\mathrm{a}, 1}$, Austin F. Murray ${ }^{\mathrm{a}}$, Dustin L. Crouch ${ }^{\mathrm{b}}, \mathrm{PhD}$, Katherine R. Saul ${ }^{\mathrm{b}}, \mathrm{PhD}$, and Jacqueline H. Cole ${ }^{\mathrm{a},{ }^{*}}, \mathrm{PhD}$

${ }^{1}$ These authors contributed equally to this work.

a Joint Department of Biomedical Engineering, University of North Carolina, Chapel Hill, NC, and North Carolina State University, Raleigh, NC, USA

${ }^{\mathrm{b}}$ Mechanical and Aerospace Engineering, North Carolina State University, Raleigh, NC, USA

\section{*Corresponding Author:}

Jacqueline H. Cole

Joint Department of Biomedical Engineering

University of North Carolina and North Carolina State University

911 Oval Drive

Campus Box 7115

Raleigh, NC 27695-7115

Tel: 919-515-5955

Fax: 919-513-3814

jacquecole@,ncsu.edu 


\section{Abstract}

2 Brachial plexus birth injury (BPBI) causes functional arm impairment in $30-40 \%$ of those affected

3 due to altered loading on the glenohumeral joint. While gross morphological osseous deformities

4 have been seen in the humerus and scapula, alterations in the underlying trabecular bone

5 microstructure and mineralization are not clear. Using a murine model of BPBI, trabecular bone

6 alterations were explored in the proximal humerus and distal scapula, which surround the

7 articulating surface of the joint. Samples were scanned using micro-CT, reoriented, and analyzed

8 for standard trabecular metrics. The regions of interest closest to the articulating surface showed

9 the greatest detriments. In the scapula, the scapular neck region showed less robust trabecular bone

10 in the neurectomy group with decreased BV/TV $(p=0.001), B M D(p=0.001)$, Conn.D $(p=0.006)$,

11 Tb.N ( $<<0.0001)$, and DA $(p=0.033)$, and increased Tb.Sp $(p<0.0001)$ compared to sham. In the

12 humerus, the epiphysis showed less robust trabecular bone in neurectomy group, but to a much

13 lesser extent than the scapular neck. The neurectomy group showed reduced BMD $(\mathrm{p}=0.007)$ and

14 Tb.N ( $\mathrm{p}=0.029)$ compared to sham. Data suggest deformities are worse near the articulating

15 surface, likely due to the greater amount of mechanical loading. The reduction in trabecular

16 microstructure and mineralization may compromise bone strength of the affected limb following

17 BPBI. Further investigation of the underlying trabecular bone deformities following injury are

18 necessary to eventually inform better treatments to limit the development of deformities.

\section{Keywords}

21 brachial plexus birth injury, trabecular microstructure, bone mineral density, glenohumeral joint 


\section{Introduction}

Functional impairment of the arm is permanent in 30-40\% of children who sustain brachial plexus birth injuries (BPBI) during difficult childbirth (Pondaag, 2004). ${ }^{(1)}$ Secondary glenohumeral dysplasia in the affected limb can result from injury (Waters 1998) and may alter the mechanical loading of the joint, which is critical for normal bone and joint development. Regions in the glenohumeral joint experience abnormal growth and macrostructural deformities after BPBI $^{(2-6)}$ (A1-Qattan 2003, Nikolaou 2011, Crouch 2014, Crouch 2015, Cheng 2015), and are comprised primarily of cancellous bone, which plays an integral role in transferring joint loading along the bone and is known to adapt to altered loads (Wolff, 1892/1986; Frost, 1994).

Trabecular bone loss has been observed in many reduced loading scenarios, such as spaceflight $^{(7)}$ (Vico et al., 2000), prolonged bedrest ${ }^{(8)}$ (Kazakia et al., 2014), and spinal cord injury (Eser et al., 2004). ${ }^{(9)}$ However, the nature and extent of trabecular bone changes after BPBI are not well understood. While no human BPBI study has examined bone microstructural properties, several studies using murine BPBI models have reported trabecular bone detriments in the humeral head, with fewer and thinner trabeculae and greater separation compared to contralateral limb and

37 sham control groups ${ }^{(10-12)}$ (Kim, 2009; Kim, 2010; Potter, , 2014). These trabecular changes were observed in conjunction with global musculoskeletal changes, including humeral anteversion and glenoid retroversion $^{(10,11)}$ (Kim et al., 2009; Kim et al., 2010), as well as decreased supraspinatus muscle volume ${ }^{(10-12)}$ (Kim et al. 2009; Kim et al., 2010; Potter et al., 2014). Beyond morphological

41 changes, the elastic modulus and overall strength of the humeral head decreased (Potter 2014). ${ }^{(12)}$

42 However, previous animal studies have not characterized trabecular changes throughout the entire glenohumeral region, particularly in the scapula glenoid region and humeral metaphysis, nor have they examined the relationship between changes in glenohumeral macrostructure and changes in 
45 the underlying trabecular microstructure. In addition, tissue mineral density is related to elastic

46 modulus, which has been seen to decrease. Yet tissue mineral density has not been characterized

47 following BPBI. This information could reveal regions of the glenohumeral joint that may be

48 especially susceptible to BPBI-induced changes in trabecular bone, ultimately informing better treatment strategies to mitigate the macrostructural deformities and address the deficits in arm

50 function experienced by human patients. throughout development (Frost, 1994; Turner, 1998). ${ }^{(13,14)}$ Altered mechanical loading has been proposed as a major contributing factor in the development of osseous deformities in the glenohumeral joint affected by BPBI. Osseous deformities reported in clinical studies include

55 flattening of the humeral and posterior subluxation relative to the scapula (Reading 2012, Waters

57 retroversion, declination, loss of glenoid concavity, and pseudoglenoid formation (Waters 1998). ${ }^{(16)}$ Computational musculoskeletal models developed from animal studies have determined that, when compared to a normal developing shoulder, the BPBI glenohumeral joint experiences 60 increased posteriorly directed, compressive loads, due to impaired growth of surrounding muscles

61 (Crouch, 2014, Cheng, 2015). ${ }^{(4,6)}$ Indications of altered mechanical loading following BPBI 62 motivate further investigation of the glenohumeral trabecular microstructure and mineralization, which bears primary responsibility for distributing loads in the shoulder joint.

The purpose of this study was to characterize microstructural features in the underlying trabecular bone near the articulating glenohumeral joint (e.g., humeral epiphysis and metaphysis, scapular regions near the glenoid) using an established rat model of BPBI (Li et al., 2008; Nikolaou

67 et al., 2015). ${ }^{(17,18)}$ Given the supporting evidence from other scenarios of altered loading and the 
68 remarkable skeletal dysplasia known to occur in clinical cases of BPBI, we hypothesized that

69 trabecular tissue mineral density and microstructure in the affected shoulder are compromised

70 during postnatal development following BPBI.

71

\section{Methods}

\section{Study Design}

This study was performed using humeri and scapulae obtained from a previous study

75 (Crouch 2015). ${ }^{(19)}$ All animal procedures were approved by the Institutional Animal Care and Use

76 Committee at the Wake Forest School of Medicine. Sixteen Sprague Dawley rat pups (Harlan

77 Laboratories, Indianapolis, Indiana) were grouped according to surgical intervention implemented

78 five days after birth: neurectomy and sham ( $n=8$ each) (Figure 1$)$.

In the neurectomy group, a postganglionic injury was surgically induced in the left forelimb

80 using an established model ( $\mathrm{Li} 2008),{ }^{(17)}$ and the right forelimb was kept completely intact as a

81 control. The rats were anesthetized with inhaled isoflurane, and a small transverse infraclavicular

82 incision was made, splitting the left pectoralis major muscle to expose the brachial plexus. The left

83 C5 and C6 nerve roots and the upper trunk of the brachial plexus were transected distal to the

84 dorsal root ganglion. The wound was irrigated with saline and closed with 6-0 nylon suture. The

85 sham group received a similar surgery with incision through the left pectoralis major muscle, but

86 the brachial plexus was not transected, and the right forelimb was kept completely intact.

After eight weeks, the rats were sacrificed, and the left (affected) and right (unaffected) scapulae and humeri were harvested. The bones were fixed in 10\% neutral buffered formalin for 
90 per group were damaged during the original dissection, leaving $n=6$ left and right scapulae and $n$

$91=7$ left and right humeri for this study.

92

$93 \quad$ Micro-Computed Tomography

Trabecular bone density and microstructure were assessed with quantitative micro-

computed tomography (micro-CT). The bones were scanned in 70\% ethanol using a $0.5-\mathrm{mm} \mathrm{Al}$

97

98

99

\section{0}

101

point of the proximal end. This VOI was positioned in the subcoracoid secondary ossification

111 center, beginning next to the articular surface and extending proximally along the scapular spine

112 axis toward the superior glenoid physis. The second scapular VOI (zone 2) was 1.5\% of the total 
113 scapular length and defined in the upper glenoid secondary ossification center, beginning inferior

114 to the superior glenoid physis and next to the articular surface extending proximally along the

115 scapular spine. The third VOI (zone 3) was 7.5\% of the total scapular length and was defined

116 within the scapular neck, beginning next to the physis running across the width of the neck and

117 extending proximally. The first humeral VOI was $12.5 \%$ of the total humeral length and defined

118 in the epiphysis, beginning inferior to the articular surface and extending distally toward the

119 proximal growth plate. The second humeral VOI was 5\% of the total humeral length and defined

120 in the metaphysis, beginning inferior to the proximal growth plate and extending distally toward

121 the diaphysis. For each VOI, standard trabecular bone metrics were calculated using the SCANCO

122 analysis software, including bone volume fraction (BV/TV), bone mineral density (BMD), tissue

123 mineral density (TMD), connectivity density (Conn.D), trabecular number (Tb.N), mean

124 trabecular thickness (Tb.Th) and separation (Tb.Sp) using direct 3D methods (Hildebrand and

125 Rüegsegger 1997), ${ }^{(21)}$ and degree of anisotropy (DA) (Bouxsein et al., 2010). ${ }^{(22)}$

$127 \quad$ Statistical Analyses

Four sets of analyses were performed. For analysis 1, limb comparisons for trabecular bone metrics were examined between affected and unaffected limbs within each group (sham, neurectomy) using paired t-tests with Welch's correction for unequal variances. For analysis 2 ,

131 group comparisons for the affected-to-unaffected ratios were examined between the neurectomy 132 and sham groups using unpaired t-tests with Welch's correction for unequal variances. For analysis 1333 , anatomical site relationships were examined between the scapula and humerus metrics using 134 linear correlations. These relationships were evaluated using both the microstructural metrics from 135 this study and the macrostructural metrics from the previous study using these same bones 
136 (humeral head width, thickness, and curvature; glenoid inclination and curvature) (Crouch

137 2015). ${ }^{(5)}$ For analysis 4, length-scale relationships between the glenohumeral joint macrostructure

138 and underlying trabecular microstructure were assessed by stepwise multiple regression with

139 forward selection, using Schwarz Bayesian information criterion to determine which trabecular

140 microstructural properties (predictor variables) best explained the variability in the macrostructural

141 measurements in the humeral head (width, thickness, and curvature) and glenoid fossa (inclination

142 and curvature). Microstructural values from the humeral epiphysis and glenoid zone 3 (Z3) were

143 used for the correlations and multiple regressions, since these regions were closest to, and thus

144 most relevant to, the articulating glenohumeral joint. Analyses 1, 2, and 3 were completed with

145 Prism 6 (GraphPad Software, Inc., La Jolla, CA), and analysis 4 was completed with SAS 9.4 (SAS

146 Institute Inc., Cary, NC). Significance was defined as $\mathrm{p}<0.05$, and trends were defined as $\mathrm{p}<$

$147 \quad 0.08$.

149 Results

150 Limb Comparisons

151 Scapula

152 For the neurectomy group, trabecular bone density and microstructure were altered in the 153 scapula of the affected limb when compared to the contralateral unaffected limb (Table 1, Figure 1543 ). Bone volume fraction was decreased 21-26\% in zones 2 and 3 and tended to decreased about $15518 \%$ in zone 1 , and bone mineral density was decreased $19-24 \%$ in zones 1,2 , and 3 , indicating a 156 reduction in bone mass on the affected side. Zone 2 also tended to show decreased tissue mineral 157 density, indicative of material change. In zones 1 and 3, trabecular number was reduced by 158 approximately $20 \%$ and trabecular separation was increased $35-44 \%$ on the affected side. Zone 2 
159 had decreased trabecular thickness by approximately $18 \%$. The alterations in trabecular 160 architecture indicate less robust trabecular bone in zones 1 and 3 on the affected side compared to 161 zone 2. In addition, zone 1 portrayed a decrease in anisotropy of about $10 \%$. zones 2 and 3 of the scapula on the affected side when compared to the contralateral limb (Table

164 1, Figure 3). Specifically, zone 2 had reduced bone volume fraction and bone mineral density by about $5 \%$, indicating decreased bone mass. In addition, zone 3 had a $12 \%$ increase in connectivity

166 density and tended to have a 5\% increase in trabecular number and 5\% decrease in trabecular

167 separation. However, the bone on the affected sides in the sham group were much less affected 168 than those in the neurectomy group.

173 density were reduced by approximately $19 \%$ and $24 \%$ respectively, revealing loss of bone mass in 174 the affected limb. Related to material changes, tissue mineral density tended to decrease by $4 \%$ on 175 the affected side. In addition, trabecular separation was increased by about $90 \%$ on the affected 176 side and trabecular number tended to decrease by $33 \%$, indicating less robust trabecular 177 architecture. In the metaphysis, less alterations were present in the affected limb (Table 2, Figure 178 4b). Trabecular material was significantly altered with a $6 \%$ reduction in tissue mineral density, 179 and connectivity density was increased by approximately $112 \%$. 

metaphysis region of the affected side portrayed increased connectivity density by $139 \%$, trabecular number by $16 \%$, and degree of anisotropy by $45 \%$, and decreased trabecular separation by $18 \%$. Trabecular thickness also tended to decrease by $6 \%$, all indicating different trabecular architecture between limbs.

Scapula

In zone 1 and 2 of the glenoid fossa region, few trabecular metrics differed significantly between the neurectomy and sham groups (Figure 4a-b). Zone 1 showed lower bone volume fraction $(-19.6 \%, \mathrm{p}=0.039)$, bone mineral density $(-19.8 \%, \mathrm{p}=0.018)$, and trabecular number $(-$ $20.6 \%, \mathrm{p}=0.028)$, and increased trabecular separation $(+38.8 \%, \mathrm{p}=0.020)$ when compared to sham. $\mathrm{p}=0.002)$, and tissue mineral density $(-3.1 \%, \mathrm{p}=0.047)$ compared to sham. Glenoid trabecular bone was most altered in zone 3 following neurectomy (Figure 4c). Bone volume fraction and bone mineral density were reduced $(-21.2 \%, \mathrm{p}=0.001 ;-22.8 \%, \mathrm{p}=0.001$ respectively $)$ compared to sham,

197 representing decreased bone mass following injury. Trabecular architecture was also less robust, 198 indicated by a decrease in connectivity density $(-28.7 \%, \mathrm{p}=0.006)$, trabecular number $(-24.5 \%$, $199 \mathrm{p}<0.0001)$, and degree of anisotropy $(-11.76 \%, \mathrm{p}=0.033)$, and an increase in trabecular separation $(+45.2 \%, \mathrm{p}<0.0001)$ 
202

203

204

205

206

207

208

209

210

211

212

213

214

215

216

217

218

219

220

221

222

Humerus

The epiphyseal region shows few altered trabecular metrics in the neurectomy group (Figure 5a). Specifically, there was a decrease in bone volume fraction $(+7.76 \%, \mathrm{p}=0.024)$, bone mineral density $(-16.3 \%, \mathrm{p}=0.007)$, and trabecular number $(-0.3 \%, \mathrm{p}=0.029)$, and a trend towards decreased connectivity density $(-34.0 \%, \mathrm{p}=0.051)$. and increased trabecular separation $(+39.4 \%$, $\mathrm{p}=0.057)$ when compared to sham. These altered metrics indicate reduction in bone mass and less robust trabecular architecture.

In the metaphyseal region, there were no significant differences between the neurectomy and sham group (Figure 5b). There were also no trends towards less robust trabecular bone in the neurectomy group compared to sham, suggesting no alterations due to injury.

\section{$\underline{\text { Anatomical Site Relationships }}$}

Correlations were present between microstructural regions near articulating glenohumeral surfaces (Table 3). Some correlations were present between microstructural regions near the articulating glenohumeral surfaces (Table 3). Tissue mineral density in scapular zone 3, representative of material properties, correlated with epiphyseal trabecular bone architecture metrics and tended to correlate with epiphyseal bone mas metrics. Specifically, scapular zone 3 tissue mineral density positively correlated with epiphyseal connectivity density ( $\mathrm{r}=0.913$, $\mathrm{p}=0.030)$ and trabecular number $(\mathrm{r}=0.946, \mathrm{p}=0.015)$, and negatively correlated with trabecular separation $(\mathrm{r}=-0.978, \mathrm{p}=0.004)$. Tissue mineral density also tended to positively correlate with bone volume fraction $(\mathrm{r}=0.837, \mathrm{p}=0.077)$ and bone mineral density $(\mathrm{r}=0.816, \mathrm{p}=0.092)$. 
In the epiphysis, degree of anisotropy correlated with some trabecular bone architecture

224 metrics and tended to correlate with bone mass metrics, all from scapular zone 3. Specifically,

225 degree of anisotropy positively correlated with zone 3 trabecular number $(r=0.973, p=0.005)$ and

226 negatively correlated with zone 3 trabecular separation $(r=-0.960, p=0.010)$. The degree of

227 anisotropy in the epiphysis also tended to positively correlate with zone 3 bone volume fraction

$228(\mathrm{r}=0.873, \mathrm{p}=0.054)$ and bone mineral density $(\mathrm{r}=0.84, \mathrm{p}=0.075)$.

$\underline{\text { Length-Scale Relationships }}$

Nearly all macrostructural bone measurements (glenoid curvature and inclination, humeral

232 head width, thickness, and curvature) were linearly correlated with at least one microstructural

233 bone metric (Table \#\#). Multiple regression models included measurements from both groups

234 (sham and neurectomy). Changes in glenoid curvature were best explained by BV/TV and TMD, 235 respectively. Glenoid inclination variability was best explained by Tb.Sp, Conn.D, Tb.N, and SMI.

236 Changes in humeral head thickness were best explained by DA, Tb.Th, TMD, and Tb.Sp. Variation

237 in humeral head curvature was best explained by Conn.D. Humeral head width did not appear to 238 be significantly affected by any particular microstructural parameter.

\section{Discussion}

The goal of this study was to investigate whether trabecular bone density and

242 microstructure are altered in regions near the glenohumeral joint following brachial plexus birth

243 injury in rats. Our findings show clear differences in the trabecular bone density and microstructure

244 for limbs affected by neurectomy compared to both their unaffected contralateral control limb and 245 sham control. The most profound differences in trabecular metrics occurred in the articulating 
246 regions of the glenohumeral joint - the humeral epiphysis and the glenoid zone 3 . The scapula was

247 affected in more parameters of trabecular bone density and microstructure relative to the humerus.

248 Our findings in the underlying trabecular bone near the glenohumeral joint provide broader context

249 for the global musculoskeletal deformities known to occur in children with BPBI.

Prior work in murine models of BPBI have reported microstructural changes to the humeral epiphysis that worsen over time, but the parameters affected differ from the current report. For example, a cross-sectional study in a CD-1 mouse model of BPBI employing the same type of neurectomy at 24 hours following birth reported a $22.2 \%$ reduction in $\mathrm{Tb}$. $\mathrm{Th}$ in the humeral epiphysis of the affected limb relative to the contralateral limb and $17.7 \%$ relative to sham at four weeks post-injury. After twelve weeks, $\mathrm{Tb}$. Th reduced further to $42.2 \%$ relative to the contralateral

256 limb and 50\% relative to sham (Kim et al., 2010). ${ }^{(13)}$ In contrast, we observed reduced epiphyseal 257 BV/TV and BMD, increased Tb.Sp, and a trend towards decreased TMD and Tb.N in the affected 258 neurectomy limb compared to the contralateral control limb and reduced BMD, Conn.D, and Tb.N compared to sham, but no significant changes to $\mathrm{Tb}$. Th. These trabecular bone losses indicate that BPBI compromises bone microstructure shortly after injury and more severely over time, suggesting that timing of post-injury treatment is critical to bone health. These results, combined with the examination of the metaphyseal region and regions of the scapula help reveal the location of the greatest disparities in affected shoulders compared to contralateral or sham shoulders.

Our group is the first to investigate the trabecular microstructure following BPBI in the 265 humeral metaphysis and regions of the scapula within the glenoid fossa. This study found that there was a significant reduction in TMD of the affected neurectomy limb compared to the 267 contralateral limb in both the epiphysis and metaphysis, and a significant reduction in TMD of the neurectomy group in the epiphysiscompared to sham. However, there were no group differences 
269 found in the metaphysis. Data suggest deformities are worse near the articulating surface, likely

270 due to the greater amount of mechanical loading. Following analyses of the humerus, this study

271 investigated the effects of BPBI on the scapula. Of the three zones, zone 3 had the least robust

272 metrics. Zone 3 resides closest to the articulating surface, also suggesting greater deformities near

273 the articulating surface. When compared to the humerus, the scapula had greater disparities,

274 especially in the trabecular architecture. The large amount of alterations in scapular microstructure

275 suggest the humerus is not affected the most post-injury.

Although the humerus was less affected than the scapula, some microstructure metrics of

277 the epiphysis did seem to correlate or trend towards a correlation with the scapular zone 3

278 microstructure metrics. The metrics most correlated were the scapular zone 3 tissue mineral

279 density with epiphyseal trabecular bone architecture metrics and bone mass metrics and the

280 epiphyseal degree of anisotropy with zone 3 trabecular bone architecture metrics and bone mass

281 metrics. This suggests that when the trabecular bone in the humeral epiphysis becomes less robust,

282 the tissue mineral density in the scapular neck is also altered. It also suggests that when the

283 trabecular bone in the scapular neck becomes less robust, the degree of anisotropy in the humeral

284 epiphysis increases, meaning the trabecular bone orientation is more anisotropic. However, the

285 trabecular metrics as a whole are not changing similarly between the two regions. Therefore, the

286 mechanical loading may be affecting one bone more than the other and not to a similar degree.

289 reductions in the tissue modulus and strength (Carter 1977), ${ }^{(23)}$ which will weaken bone's overall

290 resistance to loading. Diminished physical properties of trabeculae (e.g., BV/TV, Tb.N, Tb.Th, or

291 Tb.Sp) can also increase fracture risk, as has been shown in other clinical cases like osteoporosis ${ }^{(24)}$ 
292 (Parfitt et al., 1983) ${ }^{(38)}$ and osteopenia (Stein et al., 2014). ${ }^{(25)}$ Reduced Tb.N is a significant

293 predictor of vertebral fracture in middle-aged men diagnosed with osteopenia (Legrand et al.,

294 2000). ${ }^{(26)(40)}$ Although it has not been studied, our findings suggest that the alterations in trabecular

295 architecture following BPBI may decrease load-bearing capacity in the affected limb and increase

296 the risk of bone fracture in the humerus and scapula due to mechanical loading exceeding the

297 altered load-bearing threshold level.

298

We acknowledge that limitations exist within our study design. First, rats are weight

299 bearing quadrupeds throughout their lifetime, while human infants outgrow crawling. The different

300 relative time-periods of walking on forelimbs and hindlimbs between rats and humans may induce

301 differing mechanical loading. However, neither rats nor humans use their affected arm following

302 injury, and the murine model has been proven to produce consequences similar to humans in terms

303 of severity $(\operatorname{Li} 2010){ }^{(27)}$

In addition, this study was not powered to detect the small differences measured in

trabecular TMD and Tb.Th, but a post-hoc power analysis revealed that the measure differences

306 would require a sample size of 16 per group for TMD and 14 per group for Tb.Th to reach statistical

307 significance $(\mathrm{p}<0.05)$. While micro-CT analyses reveal trabecular bone changes following

308 neurectomy, the mechanisms underlying these changes are not well understood. Histological

309 assessments of bone remodeling may provide additional insight into the underlying mechanisms

310 driving the trabecular bone changes with peripheral nerve injury.

Finally, nerve injury is complex. Whether nerve injury directly influences skeletal

312 development remains an open question, one that is also confounded by cellular crosstalk between

313 muscle and bone cells (Hamrick, McNeil, \& Patterson, 2010). ${ }^{(28)}$ A recent study reported that

314 location of nerve injury with respect to the dorsal root ganglion can have different effects on 
315 muscle spindle preservation and longitudinal muscle growth (Nikolaou et al., 2015), ${ }^{(18)}$ which

316 motivates the need to examine these effects in more detail. Moreover, almost nothing is known

317 about the timing of nerve injury during postnatal development on the serial progression of muscle

318 and bone deficits following injury, which may provide essential information that could lead to

319 improved therapies in human patients.

321 underlying the glenohumeral joint surfaces, particularly in the scapula. While fracture more

322 commonly occurs in the clavicle as opposed to the glenohumeral bones (Ogden, 2000), ${ }^{(29)}$

323 following BPBI, our study suggests that altered trabecular bone mineralization and microstructure

324 in the glenohumeral bones may compromise bone strength during the critical period of postnatal

325 skeletal development. Longitudinal studies are necessary to assess whether increased risk of

326 fracture is an overlooked, unmet clinical need. Shoulder joint health in BPBI patients may benefit

327 from the development of better post-injury care plans that target the maintenance of underlying

328 trabecular bone. Our study is the first to characterize trabecular microstructure in the humeral

329 metaphyseal and the glenoid fossa region of the scapula, giving insight into the underlying

330 microstructure of the glenohumeral as a whole post-injury. 


\section{References}

333 1. Natural_history_of_obstetric_b.pdf.

334 2. Al-Qattan MM. Classification of Secondary Shoulder Deformities in Obstetric Brachial 335 Plexus Palsy. J. Hand Surg. 2003 Oct;28(5):483-6.

336 3. Nikolaou S, Peterson E, Kim A, Wylie C, Cornwall R. Impaired Growth of Denervated Muscle Contributes to Contracture Formation Following Neonatal Brachial Plexus Injury:

4. Crouch DL, Plate JF, Li Z, Saul KR. Computational Sensitivity Analysis to Identify Plexus Birth Palsy. J. Hand Surg. 2014 Feb;39(2):303-11.

5. Crouch DL, Hutchinson ID, Plate JF, Antoniono J, Gong H, Cao G, Li Z, Saul KR. Brachial Plexus Birth Palsy: J. Bone Jt. Surg. 2015 Aug;97(15):1264-71.

6. Cheng W, Cornwall R, Crouch DL, Li Z, Saul KR. Contributions of Muscle Imbalance and Impaired Growth to Postural and Osseous Shoulder Deformity Following Brachial Plexus bones of cosmonauts. The Lancet. 2000 May;355(9215):1607-11.

351 8. Kazakia GJ, Tjong W, Nirody JA, Burghardt AJ, Carballido-Gamio J, Patsch JM, Link T, assessed by HR-pQCT. Bone. 2014 Jun;63:132-40. 
354 9. Eser P, Schiessl H, Willnecker J. Bone loss and steady state after spinal cord injury: A

$355 \quad$ cross-sectional study using pQCT. :3.

356 10. Kim HM, Galatz LM, Patel N, Das R, Thomopoulos S. Recovery Potential After Postnatal

357 Shoulder Paralysis: An Animal Model of Neonatal Brachial Plexus Palsy. J. Bone Jt. Surg.-

358 Am. Vol. 2009 Apr;91(4):879-91.

359 11. Kim HM, Galatz LM, Das R, Patel N, Thomopoulos S. Musculoskeletal deformities

360 secondary to neurotomy of the superior trunk of the brachial plexus in neonatal mice. J.

361 Orthop. Res. 2010 Mar 11;28(10):1391-8.

362 12. Potter R, Havlioglu N, Thomopoulos S. The developing shoulder has a limited capacity to recover after a short duration of neonatal paralysis. J. Biomech. 2014 Jul;47(10):2314-20.

364 13. [Frost 1994] wolf's law.pdf.

365 14. Turner $\mathrm{CH}$. Three rules for bone adaptation to mechanical stimuli. Bone. 1998

366 Nov;23(5):399-407.

367 15. Reading BD, Laor T, Salisbury SR, Lippert WC, Cornwall R. Quantification of Humeral Head Deformity Following Neonatal Brachial Plexus Palsy: J. Bone Jt. Surg.-Am. Vol. 2012 Sep;94(18):e136-1-8.

16. Waters PM, Smith GR, Jaramillo D. Glenohumeral Deformity Secondary to Brachial Plexus Birth Palsy: J. Bone Jt. Surg. 1998 May;80(5):668-77. 
375 18. Nikolaou S, Hu L, Cornwall R. Afferent Innervation, Muscle Spindles, and Contractures

$376 \quad$ Following Neonatal Brachial Plexus Injury in a Mouse Model. J. Hand Surg. 2015

$377 \quad$ Oct;40(10):2007-16.

378 19. Crouch DL, Hutchinson ID, Plate JF, Antoniono J, Gong H, Cao G, Li Z, Saul KR.

379 Biomechanical Basis of Shoulder Osseous Deformity and Contracture in a Rat Model of

380 Brachial Plexus Birth Palsy: J. Bone Jt. Surg.-Am. Vol. 2015 Aug;97(15):1264-71.

381 20. Kothary S, Rosenberg ZS, Poncinelli LL, Kwong S. Skeletal development of the glenoid and glenoid-coracoid interface in the pediatric population: MRI features. Skeletal Radiol. 2014 Sep;43(9):1281-8.

21. Hildebrand T, Rüegsegger P. Quantification of Bone Microarchitecture with the Structure Model Index. Comput. Methods Biomech. Biomed. Engin. 1997 Jan;1(1):15-23. for assessment of bone microstructure in rodents using micro-computed tomography. J. Bone Miner. Res. 2010 Jun 7;25(7):1468-86.

23. The compressive behavior of bone as a two-phase porous structure.pdf.

24. Parfitt AM, Mathews CH, Villanueva AR, Kleerekoper M, Frame B, Rao DS. Relationships between surface, volume, and thickness of iliac trabecular bone in aging and in osteoporosis. Implications for the microanatomic and cellular mechanisms of bone loss. J. Clin. Invest. 1983 Oct;72(4):1396-409. 
399 26. Legrand E, Chappard D, Pascaretti C, Duquenne M, Krebs S, Rohmer V, Basle M-F,

400 Audran M. Trabecular bone microarchitecture, bone mineral density, and vertebral fractures

401 in male osteoporosis. J. Bone Miner. Res. 2000;15(1):13-19.

402 27. Li Z, Barnwell J, Tan J, Koman LA, Smith BP. Microcomputed Tomography

403 Characterization of Shoulder Osseous Deformity After Brachial Plexus Birth Palsy: A Rat

404 Model Study: J. Bone Jt. Surg.-Am. Vol. 2010 Nov;92(15):2583-8.

405 28. Hamrick MW, McNeil PL, Patterson SL. Role of muscle-derived growth factors in bone formation. 2013;12.

407 29. John A. Ogden. Skeletal injury in the child. 3rd ed. New York: Springer-Verlag New York;

408 2000. 
bioRxiv preprint doi: https://doi.org/10.1101/2020.02.26.967224; this version posted February 27, 2020. The copyright holder for this preprint (which was not certified by peer review) is the author/funder, who has granted bioRxiv a license to display the preprint in perpetuity. It is made available under aCC-BY 4.0 International license.

\section{Figures}

411

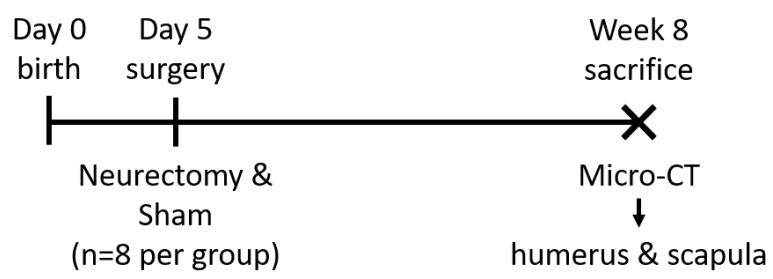

413 Figure 1. Overview of study timeline. Interventions were performed at the same timepoint.

Analyses will be performed following sacrifice at week 8 . 
a)

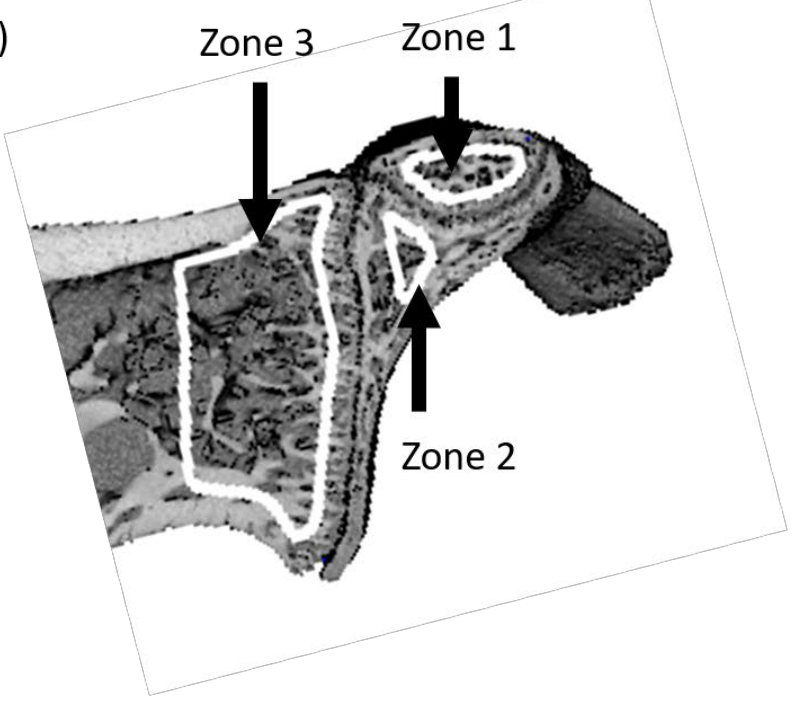

b)

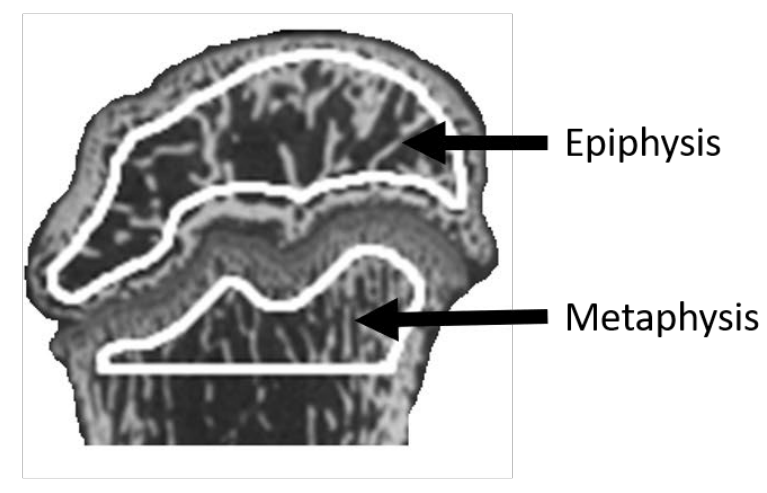

Figure 2. Trabecular bone volumes of interest (outlined in white) in the a) scapula and b) humerus, selected within different ossification centers, or zones. 
a)

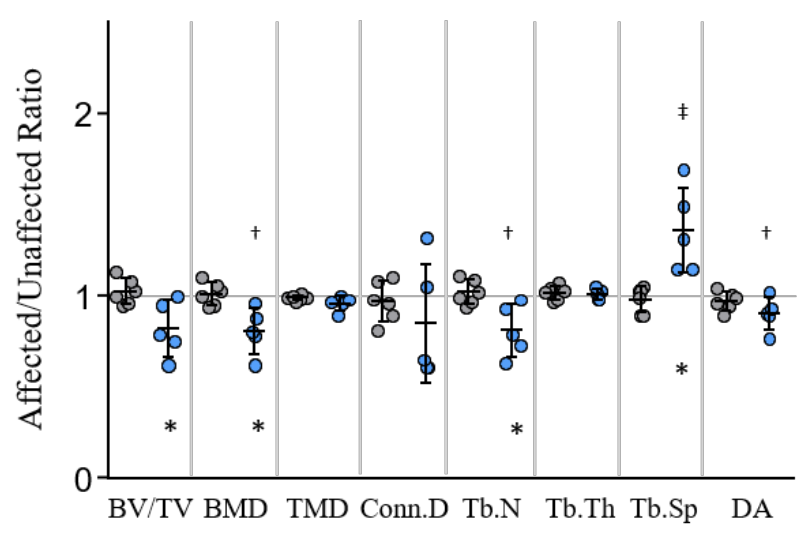

c)

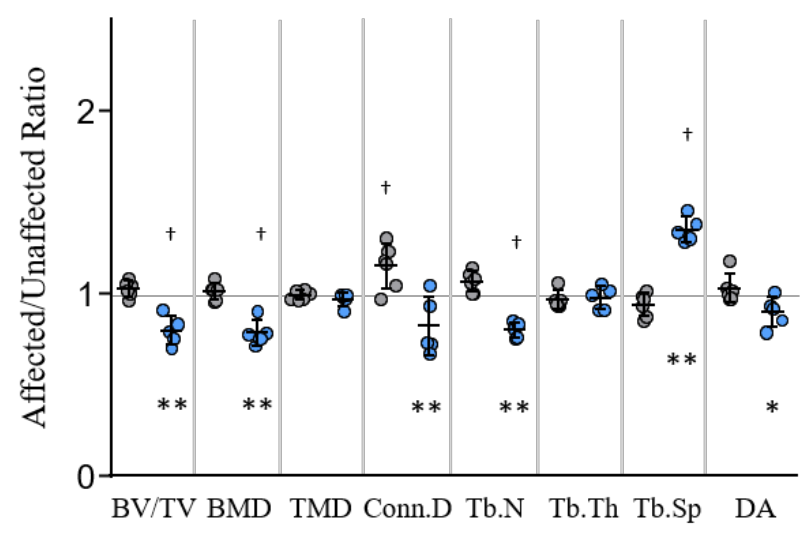

b)

Zone 2

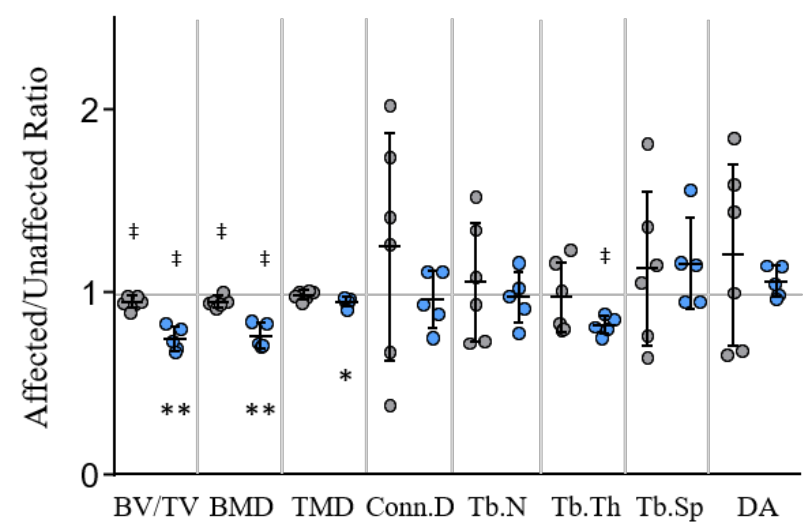

Sham

Neurectomy

Figure 3. Trabecular bone metrics in the scapula. All three zones exhibited differences in

420 trabecular microstructure in the neurectomy group with zone 3 portraying the most differences. a)

421 Zone 1 shows decreased BV/TV, BMD, and Tb.N, and increased Tb.Sp. b) Zone 2 shows

422 decreased BV/TV, BMD, and TMD. c) Zone 3 shows decreased BV/TV, BMD, Conn.D, Tb.N,

423 and DA, and increased Tb.Sp. ${ }^{\dagger} \mathrm{p}<0.05,{ }^{\star} \mathrm{p}<0.01$ affected vs. unaffected limb, ${ }^{*} \mathrm{p}<0.05,{ }^{* *} \mathrm{p}<$

4240.01 neurectomy vs. sham. 
a)

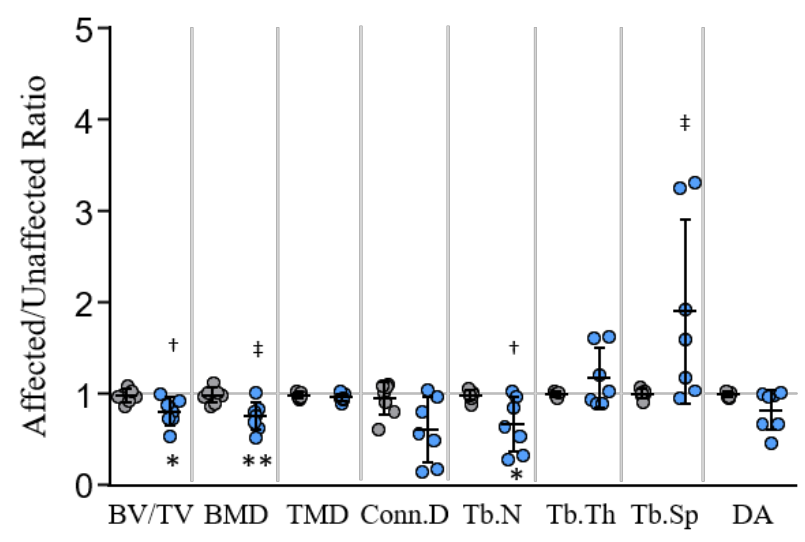

b) Metaphysis

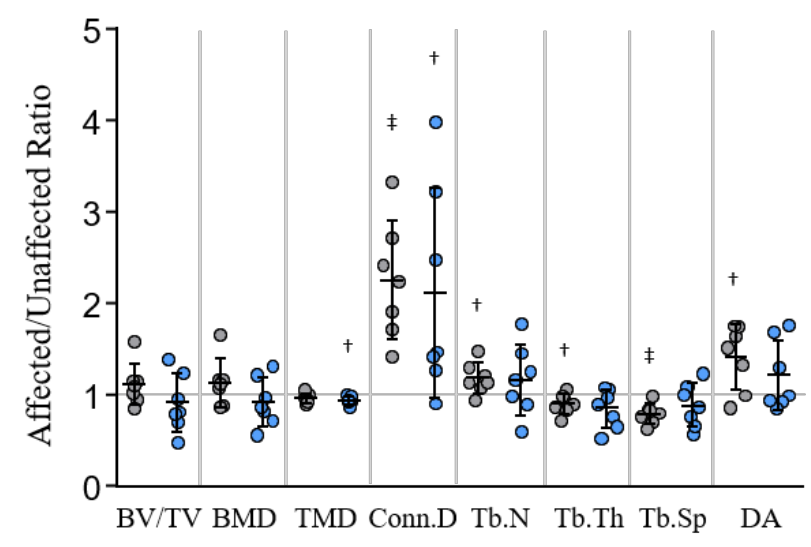

426 Figure 4. Trabecular bone metrics in the humerus. a) The epiphysis showed decreased BMD,

427 Conn.D, and Tb.N in the neurectomy group. b) The metaphysis showed no significant differences

428 between groups. ${ }^{\dagger} \mathrm{p}<0.05,{ }^{\star} \mathrm{p}<0.01$ affected vs. unaffected limb, ${ }^{*} \mathrm{p}<0.05,{ }^{* *} \mathrm{p}<0.01$ neurectomy

429 vs. sham. 


\section{Tables}

431

432 Table 1. Trabecular bone metrics in the scapula volumes of interest (VOI), expressed as percent

433 difference between the affected and the unaffected side. ${ }^{*} p<0.1,{ }^{*} p<0.05,{ }^{* *} p<0.01$ for affected

434 vs. unaffected.

\begin{tabular}{|c|c|cccccccc|}
\hline \multirow{3}{*}{ Surgery } & \multirow{2}{*}{ VOI } & \multicolumn{7}{|c|}{ Affected Side vs. Unaffected Side (\%) } \\
\cline { 3 - 10 } & & BV/TV & BMD & TMD & Conn.D & Tb.N & Tb.Th & Tb.Sp & DA \\
\hline \multirow{3}{*}{ Sham } & Zone 1 & 3.9 & 2.6 & -1.2 & -3.1 & 3.5 & 2.9 & -3.4 & -4.3 \\
& Zone 2 & $-5.4^{* *}$ & $-5.2^{* *}$ & -2.1 & 22.4 & 7.8 & -3.7 & 12.4 & 30.6 \\
& Zone 3 & 1.9 & 0.6 & -1.6 & $11.5^{*}$ & $4.8^{\#}$ & -2.8 & - & $3.0^{\#}$ \\
\hline \multirow{3}{*}{ Neurectomy } & Zone 1 & $-17.8^{\#}$ & $-19.4^{*}$ & -3.9 & -15.2 & $-18.8^{*}$ & 0.9 & $44.0^{* *}$ & $-9.7^{*}$ \\
& Zone 2 & $-25.6^{* *}$ & $-23.9^{* *}$ & $-5.3^{\#}$ & -4.3 & -3.0 & $-18.3^{* *}$ & 15.6 & 5.6 \\
& Zone 3 & $-20.5^{*}$ & $-21.8^{*}$ & -3.5 & $-18.2^{\#}$ & $-20.1^{*}$ & -2.6 & $34.8^{*}$ & -10.2 \\
\hline
\end{tabular}


436 Table 2. Trabecular bone metrics in the humerus volumes of interest (VOI), expressed as percent

437 difference between the affected and the unaffected side. ${ }^{*} p<0.1,{ }^{*} p<0.05,{ }^{*} \mathrm{p}<0.01$ for affected

438 vs. unaffected.

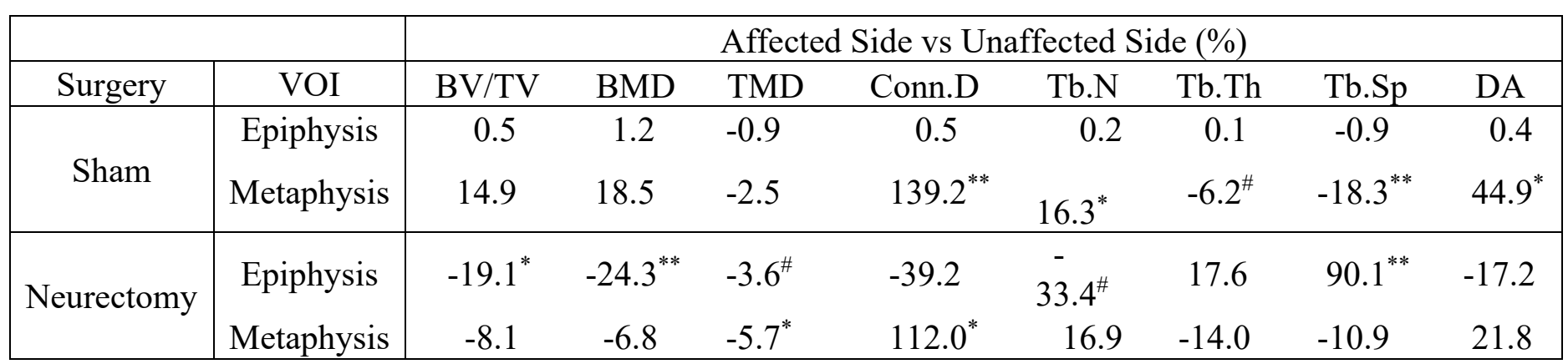


440 Table 3. Pearson's correlation coefficients (r) and associated p-values for correlations of trabecular

441 density and microstructure of the epiphysis and scapular zone 3 in the neurectomy groups. $p<0.05$

442 in bold.

Zone3

\begin{tabular}{|c|c|c|c|c|c|c|c|c|c|c|}
\hline & & & \\
\hline & & & $\mathrm{BV} / \mathrm{TV}$ & BMD & TMD & ConnD & Tb.N & Tb.Th & Tb.Sp & DA \\
\hline \multirow{16}{*}{ Epiphysis } & \multirow{2}{*}{$\mathrm{BV} / \mathrm{TV}$} & $r$ & -0.049 & 0.148 & 0.837 & 0.005 & -0.270 & -0.275 & 0.349 & -0.506 \\
\hline & & $\mathrm{p}$ & 0.938 & 0.812 & 0.077 & 0.994 & 0.660 & 0.655 & 0.565 & 0.385 \\
\hline & \multirow{2}{*}{ BMD } & $\mathrm{r}$ & -0.079 & 0.115 & 0.816 & 0.059 & -0.280 & -0.384 & 0.380 & -0.554 \\
\hline & & $\mathrm{p}$ & 0.900 & 0.854 & 0.092 & 0.925 & 0.646 & 0.523 & 0.528 & 0.333 \\
\hline & \multirow{2}{*}{ TMD } & $\mathrm{r}$ & -0.055 & 0.082 & 0.721 & 0.250 & 0.043 & -0.618 & 0.081 & -0.493 \\
\hline & & $\mathrm{p}$ & 0.930 & 0.895 & 0.169 & 0.685 & 0.946 & 0.266 & 0.897 & 0.399 \\
\hline & \multirow{2}{*}{ ConnD } & $\mathrm{r}$ & 0.097 & 0.285 & 0.913 & 0.170 & -0.090 & -0.309 & 0.185 & -0.395 \\
\hline & & $\mathrm{p}$ & 0.877 & 0.642 & 0.030 & 0.785 & 0.888 & 0.613 & 0.766 & 0.512 \\
\hline & \multirow{2}{*}{ Tb.N } & $\mathrm{r}$ & 0.247 & 0.428 & 0.946 & 0.311 & 0.005 & -0.280 & 0.105 & -0.266 \\
\hline & & $\mathrm{p}$ & 0.688 & 0.472 & 0.015 & 0.610 & 0.994 & 0.648 & 0.867 & 0.665 \\
\hline & \multirow{2}{*}{ Tb.Th } & $\mathrm{r}$ & 0.070 & 0.248 & 0.696 & -0.130 & -0.360 & 0.135 & 0.377 & -0.263 \\
\hline & & $\mathrm{p}$ & 0.912 & 0.688 & 0.192 & 0.839 & 0.553 & 0.828 & 0.532 & 0.669 \\
\hline & \multirow{2}{*}{ Tb.Sp } & $\mathrm{r}$ & -0.356 & -0.525 & -0.978 & -0.400 & -0.140 & 0.251 & 0.035 & 0.157 \\
\hline & & $\mathrm{p}$ & 0.557 & 0.363 & 0.004 & 0.509 & 0.817 & 0.684 & 0.956 & 0.801 \\
\hline & \multirow{2}{*}{$\mathrm{DA}$} & $\mathrm{r}$ & 0.873 & 0.840 & 0.462 & 0.648 & 0.973 & 0.250 & -0.960 & 0.766 \\
\hline & & $\mathrm{p}$ & 0.054 & 0.075 & 0.433 & 0.237 & 0.005 & 0.685 & 0.010 & 0.131 \\
\hline
\end{tabular}


Table 4. Pearson's correlation coefficients (r) and associated p-values for correlations of trabecular density and microstructure with

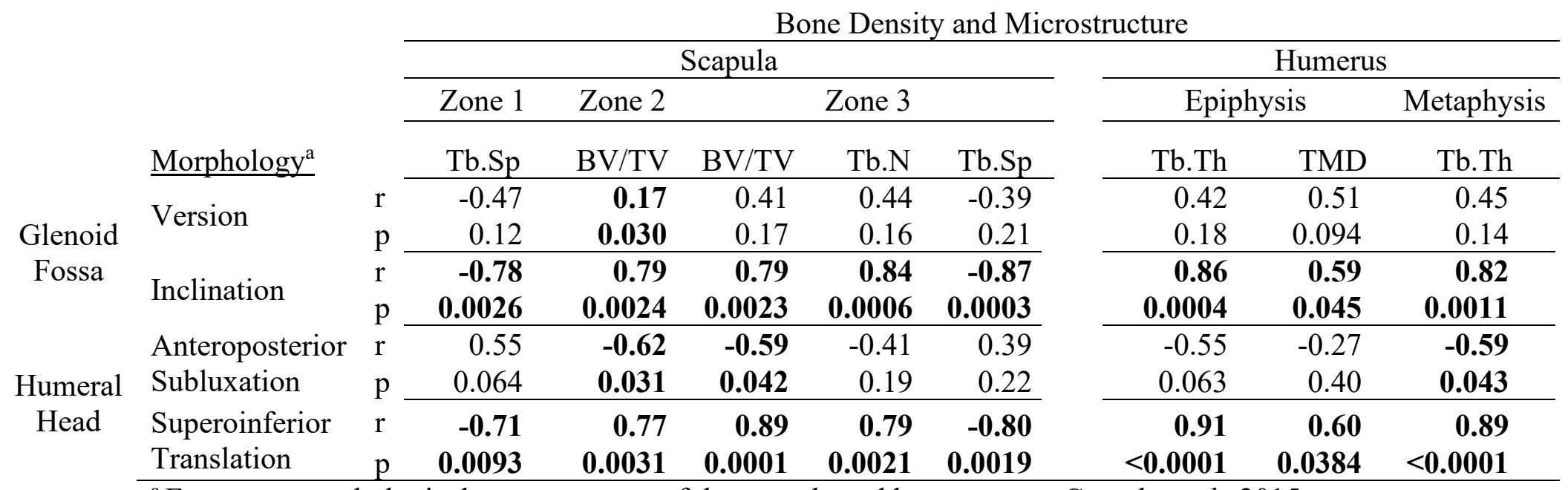

${ }^{\mathrm{a}}$ For gross morphological measurements of the scapula and humerus, see Crouch et al., 2015. 